\title{
Material parameters for electrostriction
}

Yuri M. Shkel and Daniel J. Klingenberga)

Department of Chemical Engineering and Rheology Research Center, University of Wisconsin, Madison, Wisconsin 53706

(Received 11 March 1996; accepted for publication 15 July 1996)

Electrostriction is often described by a phenomenological tensor relating a material's deformation to an applied electric field. However, this tensor is not a material parameter; for deformable, weakly compressible materials (e.g., elastomers), the field-induced deformations depend strongly upon boundary conditions. A different approach that relates the deformation to material properties as well as boundary conditions is required. In this article, we describe a linear theory which introduces five material parameters governing electrostriction: the relative dielectric constant, $\epsilon^{0}$, two derivatives of the dielectric constant tensor, $a_{1}$ and $a_{2}$, Young's modulus, $E_{y}$ and Poisson's ratio, $\nu$. Knowledge of these parameters and appropriate boundary conditions allow one to predict field-induced deformations for arbitrary configurations. We demonstrate an experimental procedure for measuring deformations and permittivity changes, from which the parameters $a_{1}$ and $a_{2}$ may be extracted $\left(\epsilon^{0}, \nu\right.$, and $E_{y}$ can be measured by a variety of established methods). The linear theory reproduces experimental results for two polyurethane films at small to moderate electric field strengths. We find that the electrostatic force associated with the parameters $a_{1}$ and $a_{2}$ is at least ten times larger than the Coulombic attractive force between the electrodes. (C) 1996 American Institute of Physics. [S0021-8979(96)05920-8]

\section{INTRODUCTION}

The elastic deformation of a dielectric material under the forces exerted by an electrostatic field is called electrostriction. ${ }^{1}$ Electrostriction is traditionally described by a tensor of electrostrictive coefficients, $\gamma_{i j k l}$, linking the strain tensor $u_{i j}\left[=(1 / 2)\left(\partial u_{i} / \partial x_{j}+\partial u_{j} / \partial x_{i}\right)\right.$, where $\mathbf{u}(\mathbf{x})$ is the displacement field] with the components of the electric field $\mathbf{E},{ }^{2}$

$$
u_{i j}=\gamma_{i j k l} E_{k} E_{l} .
$$

Employing such an approach appears natural and convenient for applications, and has been examined in a variety of studies. ${ }^{3-5}$ In order to demonstrate limitations of such an approach, let us consider a general description of electrostrictive deformation.

Mechanical equilibrium of an electrostrictive material is described by

$$
\nabla \cdot\left(\boldsymbol{\sigma}^{\text {elast. }}+\boldsymbol{\sigma}^{\text {elect. }}\right)=\mathbf{0},
$$

where the total stress in the medium $\boldsymbol{\sigma}$ is a sum of the elastic stress tensor $\boldsymbol{\sigma}^{\text {elast. }}$ and the electrostatic (Maxwell) stress tensor $\boldsymbol{\sigma}^{\text {elect. }}$. Determining the deformation requires solving Maxwell's equations for the electrostatic fields, $\nabla \cdot \mathbf{D}=0$ and $\nabla \times \mathbf{E}=0$, simultaneously with Eq. (2), subject to the appropriate boundary conditions. This process requires specifying constitutive equations relating the elastic and electrostatic stresses to material properties and thermodynamic variables. In the following section, examples of such constitutive equations for linear materials are presented.

It is possible to make general statements that do not depend on the particular forms of these constitutive equations. First, the electrostatic stress and its effect on the de-

${ }^{a)}$ Electronic mail: klingen@neep.engr.wisc.edu formation depends on the field distribution and therefore upon the electrode configuration (e.g., shape and boundary conditions) and the electrical properties of the medium. The elastic stresses, in general, will also depend upon the conditions at the material boundaries and perhaps other features such as the sample history. Thus $\gamma_{i j k l}$ may be determined by solving Eq. (2), assuming that the constitutive equations and the associated parameters, as well as the boundary conditions, are known.

It should be recognized, however, that since the deformation depends on the conditions at the material's boundaries, $\gamma_{i j k l}$ is not a material property in the strictest definition. $\gamma_{i j k l}$ measured for one particular set of conditions will not necessarily describe the behavior under a different set of conditions. This distinction is not so important for inorganic electroactive materials with large Young's moduli-the elastic properties of attached fixtures (e.g., electrodes) are relatively unimportant and the tensor $\gamma_{i j k l}$ does not vary between different configurations. Furthermore, many applications employ similar shapes. In contrast, polymers are often more flexible than the bounding materials, and thus the deformation for a given electric field strength can vary considerably from one configuration to another. In this situation, a set of electrostriction coefficients as defined above is not sufficient to describe the field-induced deformation of the material.

In this article, we employ a linear theory to relate properties of a material, which do not depend on the configuration, to the electric field-induced deformation. The forces generated by the electric field are determined by three material parameters - the permittivity of the polymer in the absence of deformation, $\epsilon^{0}$, and two derivatives of the dielectric tensor with respect to strain components, $a_{1}$ and $a_{2}$. The deformation resulting from these forces is described by Hooke's law, which adds two additional material properties (e.g., Young's modulus, $E_{y}$, and Poisson's ratio, $\nu$ ). Thus 
five material properties, measurable in independent tests, are sufficient to completely describe the deformation induced by an applied electric field for arbitrary configurations. As this linear theory describes relatively idealized (linear) materials, a minimum of five material parameters are required to describe electrostriction in general.

An experimental technique, based upon measuring the permittivity change of a material due to deformation, is described for the study of electrostrictive deformation of two thin polymer films. This procedure provides estimates of the dielectric coefficients $a_{1}$ and $a_{2}$ at the experimental temperature and field frequency. The results show that under certain limitations, the linear theory of electrostriction is valid for these materials. Thus, assuming that the remaining parameters (permittivity, Young's modulus, and Poisson's ratio) are known or can be measured by standard methods, the electrostrictive deformation of these polymeric materials may be calculated for arbitrary configurations.

\section{LINEAR THEORY OF ELECTROSTRICTION FOR ISOTROPIC MATERIALS}

A linear theory for electrostriction, appropriate for small deformations, may be developed using simplifying assumptions:

- the dielectric properties of the unstressed material are isotropic;

- the set of the thermodynamic parameters, the temperature $T$, the strain tensor $u_{i k}$, and the magnitude of an external electric field $E$, are sufficient to specify the state of the system (e.g., transients are not considered);

- the relationships between the mechanical stress and strain tensors are given by Hooke's law; and

- the dielectric displacement is linear in the electric field, $D_{i}=\epsilon_{0} \epsilon_{i j} E_{j}$, where $\epsilon_{0}$ is the permittivity of free space.

These assumptions lead to the relationship between the dielectric and strain tensors, the electrostatic (Maxwell) stress tensor, which describes the forces induced by the electric field, and the resulting deformation of a linear elastic material (provided necessary boundary conditions are specified).

A stressed material is anisotropic and its dielectric properties must be described by a dielectric tensor. The most general dielectric tensor of rank two which is linear in the components of the strain tensor $u_{i k}$ is

$$
\epsilon_{i j}=\epsilon^{0} \delta_{i j}+a_{1} u_{i j}+a_{2} u_{k k} \delta_{i j} .
$$

$\epsilon^{0}$ is the relative dielectric constant of the undeformed, isotropic body and the other two terms describe the dielectric properties of the deformed material. ${ }^{1,6,7}$ The coefficients $a_{1}$ and $a_{2}$ in this equation are functions of the thermodynamic parameters, the temperature $T$ and the external field magnitude $E$, as well as the field frequency, $f$.

Forces produced by the electric field may be expressed through Maxwell's stress tensor, ${ }^{1,6}$

$$
\sigma_{i j}^{\text {elect. }}=\epsilon_{0} \frac{2 \epsilon^{0}-a_{1}}{2} E_{i} E_{j}-\epsilon_{0} \frac{\epsilon^{0}+a_{2}}{2} E^{2} \delta_{i j}
$$

For a linear elastic material, the elastic stress is described by Hooke's law, a)

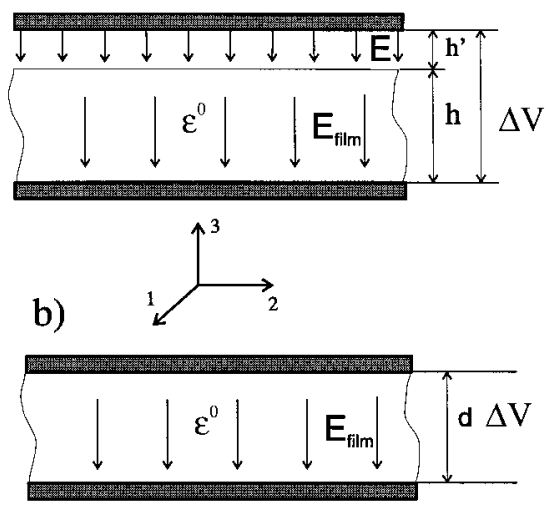

FIG. 1. Schematic diagram illustrating the definitions of electric fields in and near a thin polymer film placed between two parallel electrodes in two different configurations (neglecting edge effects). (a) $E=\epsilon^{0} E_{\text {film }}$, and $E_{\text {app }}=\Delta V /\left(h+h^{\prime}\right)=\left(E h^{\prime}+E_{\text {film }} h\right) /\left(h+h^{\prime}\right)$, where $E_{\text {app }}$ is the apparent field strength; (b) $E=\epsilon^{0} E_{\text {film }}$, and $E_{\text {app }}=\Delta V / h=E_{\text {film }}$.

$$
\sigma_{i j}^{\text {elast. }}=\frac{E_{y}}{1+\nu}\left(u_{i j}+\frac{\nu}{1-2 \nu} u_{k k} \delta_{i j}\right)
$$

where Young's modulus, $E_{y}$, and Poisson's ratio, $\nu$, are the elastic parameters of the medium. ${ }^{8}$

Thus for this simple model of linear electrostrictive materials, the problem of electrostrictive deformation is associated with five material parameters: $\epsilon^{0}, a_{1}, a_{2}, E_{y}$, and $\nu$. As discussed in the previous section, electrostrictive deformation can now be determined by simultaneous solution of Eq. (2) and Maxwell's equations, along with appropriate boundary conditions. For uniform electric fields, this process simplifies considerably because $\nabla \cdot \boldsymbol{\sigma}^{\text {elect. }}=\mathbf{0}$ and the electric field appears only in the boundary conditions for mechanical equilibrium.

A convenient configuration for experimental study is a plane dielectric film placed between parallel-plate electrodes where the electric field is indeed uniform (neglecting edge effects). Even with this simple geometry, the field-induced deformations can vary considerably, depending on the manner in which the film is placed between the electrodes. A few special cases are considered below.

Consider the two situations depicted in Fig. 1, where a thin film is placed parallel between two parallel electrodes. In Fig. 1(a), there is an air gap between the polymer and an electrode. The electric field in the air is $E\left(-\boldsymbol{\delta}_{3}\right)=\epsilon^{0} E_{\text {film }}\left(-\boldsymbol{\delta}_{3}\right)$, where $E_{\text {film }}$ is the magnitude of the field within the film. The net force per unit area, induced by the electric field and directed into the film, can be determined from Eq. (4),

$$
f_{n}=\epsilon_{0}\left[\frac{1-\epsilon^{0}}{\epsilon^{0}}-\frac{a_{1}+a_{2}}{\left(\epsilon^{0}\right)^{2}}\right] \frac{E^{2}}{2} .
$$

In Fig. 1(b), the film is in intimate contact with both electrodes, and the resulting force per unit area directed into the polymer is 


$$
f_{n}=\epsilon_{0}\left[\frac{1}{\epsilon^{0}}-\frac{a_{1}+a_{2}}{\left(\epsilon^{0}\right)^{2}}\right] \frac{E^{2}}{2},
$$

where $E=\epsilon^{0} E_{\text {film }}$ is the external field strength that would produce a field strength of $E_{\text {film }}$ within the film.

The difference between the forces [Eq. (7)-Eq. (6)] is $\epsilon_{0} E^{2} / 2$, which equals the Coulombic attraction between the charged electrodes. This extra force, although contributing to the deformation of a film in contact, has nothing to do with electrostriction-this is an electric force exerted on the electrodes, which is transmitted mechanically to the film.

The deformation resulting from these forces is determined from Eqs. (2) and (5) $\left(\nabla \cdot \boldsymbol{\sigma}^{\text {elect. }}=\mathbf{0}\right)$, along with appropriate boundary conditions. Of primary concern for electrostrictive films is the change in thickness, characterized by the deformation

$$
u_{33}=-\frac{f_{n}}{g\left(E_{y}, \nu, \ldots\right)},
$$

where $g\left(E_{y}, \nu, \ldots\right)$ is the effective film stiffness which depends on $E_{y}, \nu$, and the mechanical boundary conditions. Here we will consider two limiting cases. For an nonconstrained film in which the shear stress is zero at the film/ electrode interface, and for zero normal and shear stresses at the film edges, the effective stiffness is ${ }^{8}$

$$
g\left(E_{y}, \nu, \ldots\right)=E_{y} .
$$

Such a situation may be realized if the film material can slip freely at the electrodes, or if the electrodes are sufficiently deformable.

For a constrained film in which the material in contact with a rigid electrode cannot translate relative to the interface (or for which the edges cannot translate), the effective stiffness is ${ }^{8}$

$$
g\left(E_{y}, \nu, \ldots\right)=\frac{E_{y}(1-\nu)}{(1+\nu)(1-2 \nu)} .
$$

For incompressible materials $(\nu=0.5)$, there will be no deformation. As many polymer solids have Poisson's ratios very close to 0.5 (e.g., 0.499), ${ }^{9}$ deformations when constrained will be very small. Note that both of the above equations may be written as $u_{33}=\gamma E_{\text {film }}^{2}$, but the coefficient $\gamma$ depends on the mechanical boundary conditions, the location of the electrodes relative to the polymer surfaces (e.g., see Eqs. (6) and (7)), as well as five material properties.

In both of the above cases, the magnitude of the deformation depends on the values of the dielectric parameters $a_{1}$ and $a_{2}$ (e.g., through Eq. (6) or (7)). From Eq. (3), the relationship between these parameters and the change in $\epsilon_{33}$ with $u_{33}$ is

$$
a_{1}+a_{2} \frac{d u_{k k}}{d u_{33}}=\frac{d \epsilon_{33}}{d u_{33}} .
$$

Thus the parameters $a_{1}$ and $a_{2}$ can be determined from two measurements in which $d u_{k k} / d u_{33}$ differs. For example, for uniform deformation of an nonconstrained plane film, Eq. (11) becomes

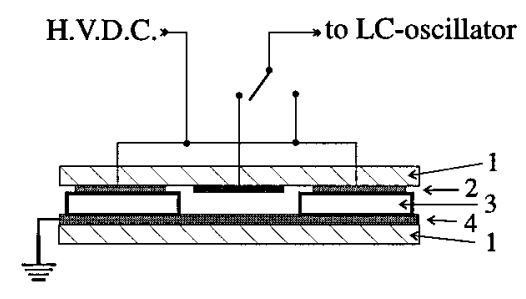

FIG. 2. Schematic diagram of the polymer-air-polymer capacitor assembly (not to scale). The different layers are: 1-glass slides; 2-three-electrode system; 3-polymer films and air gap; and 4-ground electrode.

$$
a_{1}+a_{2}(1-2 \nu)=\frac{d \epsilon_{33}}{d u_{33}},
$$

while for uniform deformation of a constrained plane film,

$$
a_{1}+a_{2}=\frac{d \epsilon_{33}}{d u_{33}} .
$$

Thus, if $d \epsilon_{33} / d u_{33}$ can be measured in both of the above configurations, $a_{1}$ and $a_{2}$ can be determined by simultaneously solving Eqs. (12) and (13).

We conclude the presentation of this linear model by reiterating a few important points. Field-induced deformations vary quadratically with the electric field strength, and the proportionality constant depends upon five material properties $\left(\epsilon^{0}, a_{1}, a_{2}, E_{y}\right.$, and $\left.\nu\right)$ as well as the conditions at the material boundaries. The proportionality constant itself is not a material parameter. Although the theory is developed to describe the deformation of elastic materials under large electric fields $\left(\geqslant 10^{6} \mathrm{~V} / \mathrm{m}\right)$, all five parameters may be determined from experiments under small or no electric fields.

In the remainder of this report, we describe an experimental technique for measuring the field-induced deformation and the resulting change in permittivity of electrostrictive polymer films. The deformations are quadratic in the applied field strength for small to moderate field strengths, but they deviate from the linear theory at larger field strengths. The permittivity changes also agree with the model for small to moderate field strengths, varying linearly with the deformation and quadratically with the electric field strength. The parameters $a_{1}$ and $a_{2}$ are estimated by measuring the quantity defined in Eq. (11) under conditions where $d u_{k k} / d u_{33}$ varies. The results indicate that the electrostatic forces associated with $a_{1}$ and $a_{2}$ are significantly larger than the Coulombic attraction between the electrodes.

\section{EXPERIMENT}

Deformation and changes in permittivity of polymer film samples were determined by measuring the change in capacitance of a composite polymer-air-polymer capacitor. The sample capacitor is contained between glass plates (Fig. 2). One glass plate is attached to a large grounded copper electrode, and the second glass plate is attached to three separate copper electrodes, each $0.75 \times 2.0 \mathrm{in}^{2}$. The two outer electrodes are maintained at the same electric potential. Two polymer films, obtained by cutting a single film in half, are placed between each of the outer plates and the ground elec- 
trode. These two polymer films, which constitute a single polymer-film capacitor, act as a spacer for the center air-gap capacitor. Experiments are conducted by applying a large potential difference across the outer electrodes and the ground electrode, then measuring separately the change capacitance of the polymer-film and air-gap capacitors.

The capacitance $C$ of a plane capacitor with plate area $A$ and gap $h \ll \sqrt{A}$ is ${ }^{6}$

$$
C=\frac{\epsilon_{0} \epsilon A}{h}\left[1+O\left(\frac{\log (\sqrt{A} / h)}{2 \sqrt{A} / h}\right)\right] .
$$

For the results reported here, edge effects are small, and the second term in the brackets is less than $1 \%$ of the total capacitance and can be ignored. For the air-gap capacitor, $\epsilon \approx 1$.

Application of an electric field produces a small capacitance change for both the air-gap capacitor $\left(C_{\text {air }}\right)$ and the polymer film capacitor $\left(C_{\text {poly }}\right)$. For the air-gap capacitor, the change arises from only the change in the gap,

$$
\frac{\delta C_{\text {air }}}{C_{\text {air }}^{0}}=-\frac{\delta h}{h^{0}},
$$

where the superscript 0 refers to the reference state from which the change was measured. For the polymer-film capacitor, the capacitance change contains contributions from the gap change as well as the permittivity change,

$$
\frac{\delta C_{\text {poly }}}{C_{\text {poly }}^{0}}=-\frac{\delta h}{h^{0}}+\frac{\delta \epsilon}{\epsilon^{0}} .
$$

Since the gap change for both capacitors is the same, measurement of $\delta C_{\text {air }} / C_{\text {air }}^{0}$ and $\delta C_{\text {poly }} / C_{\text {poly }}^{0}$ provides $\delta h / h^{0}$ as well as $\delta \epsilon / \epsilon^{0}$. For small deformations, $u_{33} \approx \delta h / h^{0}$ and $\left(d \epsilon_{33} / d u_{33}\right) / \epsilon^{0} \approx\left(\delta \epsilon / \epsilon^{0}\right) /\left(\delta h / h^{0}\right)$, and thus the dielectric parameters are related to the capacitance changes by

$$
\begin{aligned}
\frac{1}{\epsilon^{0}} \frac{d \epsilon_{33}}{d u_{33}} & =\frac{1}{\epsilon^{0}}\left(a_{1}+a_{2} \frac{d u_{k k}}{d u_{33}}\right) \\
& =-\frac{\delta C_{\mathrm{poly}} / C_{\mathrm{poly}}^{0}-\delta C_{\mathrm{air}} / C_{\mathrm{air}}^{0}}{\delta C_{\mathrm{air}} / C_{\mathrm{air}}^{0}} .
\end{aligned}
$$

A schematic diagram of the measurement apparatus is presented in Fig. 3. Capacitance changes were determined by measuring the frequency change of a Hartley $(L C)$ oscillator circuit which included the sample capacitor in series with a high-voltage blocking capacitor. Application of a similar $L C$ oscillator to measure small inductance variations has been discussed in Ref. 10. The oscillation frequency $f$ depends on the inductance, $L$, and equivalent capacitance, $C$, of the circuit. If the inductance is constant during the test (i.e., independent of deformation and electric field strength), a variation of the frequency is a result of a variation of the capacitance $C$. The dc current was measured during some experiments with a Keithley 4853 picoammeter to verify that the film conductivity did not increase significantly during the tests. Therefore, variations of the sample capacitance $\delta C$ are related to the frequency change $\delta f$ by

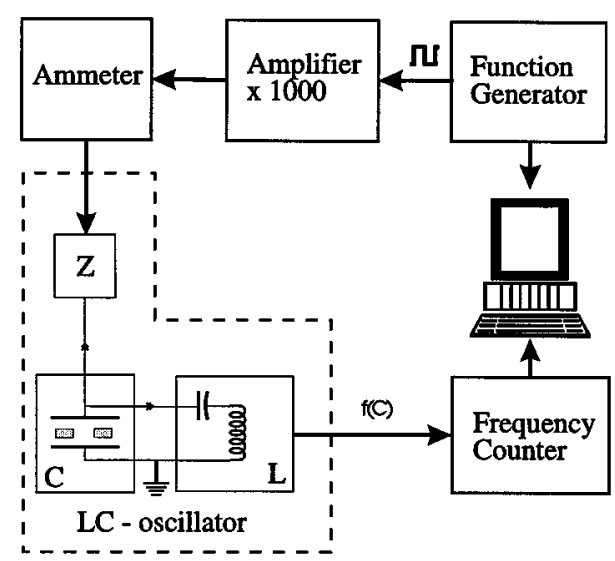

FIG. 3. Schematic diagram of the measurement apparatus. The system measures the change in oscillator frequency due to a capacitance change arising from the film deformation. A large impedance $(Z)$ isolates the oscillator from the ammeter and amplifier.

$$
\frac{\delta C}{C^{0}}=-\beta \frac{\delta f}{f^{0}},
$$

where, again, the superscript " 0 " refers to the reference state from which changes were measured. The coefficient $\beta$ $(\approx 3$.) depends on the Hartley oscillator and the sample capacitance relative to the equivalent capacitance; this parameter is constant during measurements and determined by calibration. Frequency changes were measured with a Keithley PCIP-CNTR Counter/Timer.

Large potential differences were supplied to the polymer-film capacitors by a function generator (Stanford Research Systems, model DS345), amplified with a Trek (model 10/10) amplifier. A unipolar square wave signal with a period of $40 \mathrm{~s}$ was employed, with amplitudes in the range of 50-4000 V. The capacitances of the air-gap and polymer film capacitors were in the range of $30-300 \mathrm{pF}$, resulting in an oscillator frequency in the range $0.5-1.5 \mathrm{MHz}$. The amplitude of this high frequency voltage was $\approx 2 \mathrm{~V}$.

Two types of polyurethane films (JPS Elastomer Corp.) were examined in this study. MP1880 was a polyether with a thickness of 0.020 in. $\left(5.0 \times 10^{-4} \mathrm{~m}\right)$, and the other, MP1495, was a polyester with a thickness of 0.003 in. $\left(7.6 \times 10^{-5} \mathrm{~m}\right)$. These films were visually smooth. Thickness measurements over the electroded area, measured with a micrometer (precision \pm 0.0005 in.), did not reveal any measurable variations. When placed between the copper electrodes, these films apparently achieved good contact between the metal surfaces as they offered significant resistance when we subsequently removed them. It is clear that the interfaces between the polymer and the electrodes are very important and deserve more attention than we have given them to this point. This issue is discussed further below.

\section{RESULTS AND DISCUSSION}

The deformation $\left(-\delta h / h^{0}\right)$ of both polymer films is presented as a function of applied electric field strength in Fig. 4. The deformations were determined from the change in oscillator frequency, as described above, immediately af- 


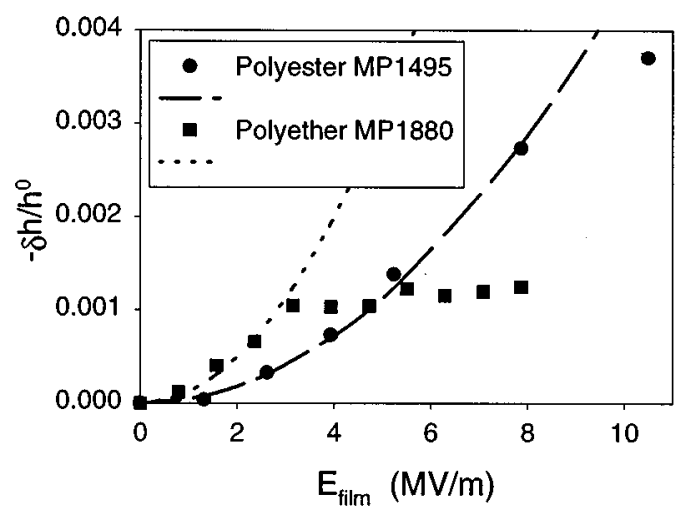

FIG. 4. Deformation $\left(-\delta h / h^{0}\right)$ as a function of electric field strength $\left(E_{\text {film }}=E_{\text {app }}\right)$ for two polyurethane films. The symbols are the experimental data and the curves are parabolic fits to the small field strength data. For the MP1495 data, $\left(-\delta h / h^{0}\right) / E_{\text {film }}^{2}=4.5 \times 10^{-17}(\mathrm{~m} / \mathrm{V})^{2}$, and for the MP1880 data, $\left(-\delta h / h^{0}\right) / E_{\text {film }}^{2}=1.3 \times 10^{-16}(\mathrm{~m} / \mathrm{V})^{2}$.

ter the electric field was applied, using the state immediately preceding the field application as the reference. The materials continue to deform for a long time after the field is applied or removed. The results reported here reflect the short time (high frequency) responses. The deformation of viscoelastic materials will be examined in future studies.

$-\delta h / h^{0}$ increases approximately quadratically with electric field strength at low field strengths for both polymers, in agreement with the linear theory. At larger field strengths, the deformation increases more slowly. We have identified three possible explanations for this behavior. First, the deformation may be mechanically nonlinear (i.e., Hooke's law no longer applies); second, the response may be dielectrically nonlinear (i.e., in this regime, $\epsilon^{0}, a_{1}$, and $a_{2}$ may all depend on field strength); or third, the conditions at the polymer-electrode interfaces may change-for example, from a state at low electric fields where friction is minimal and the polymer can slide freely across the electrodes, to a state at large electric fields where friction is more significant and the polymer deformation is constrained.

$\delta \epsilon / \epsilon^{0}$ for the same experiments is plotted as a function of electric field strength in Fig. 5. The data represent the change in permittivity immediately following the application of the electric field, with the state immediately preceding the field application as the reference. As with the deformation, the permittivity continues to change slowly at long times after applying or removing the electric field. Again, these long time transients will be examined in future studies, but will not be discussed further here. $\delta \epsilon / \epsilon^{0}$ increases quadratically with electric field strength at low field strengths, and thus varies linearly with the deformation as described by Eq. (3). At large field strengths, the permittivity change saturates, perhaps due to one of the mechanisms listed above. We note that the relative changes in permittivity are significantly larger than the relative deformations for both polymers.

$a_{1}$ and $a_{2}$ may be estimated by intentionally varying the conditions at the film-electrode interfaces. This goal is achieved by measuring $\delta h / h^{0}$ and $\delta \epsilon / \epsilon^{0}$ due to a relatively small applied electric field of $4 \times 10^{6} \mathrm{~V} / \mathrm{m}$, with additional weights placed on top of the measurement assembly (in ad-

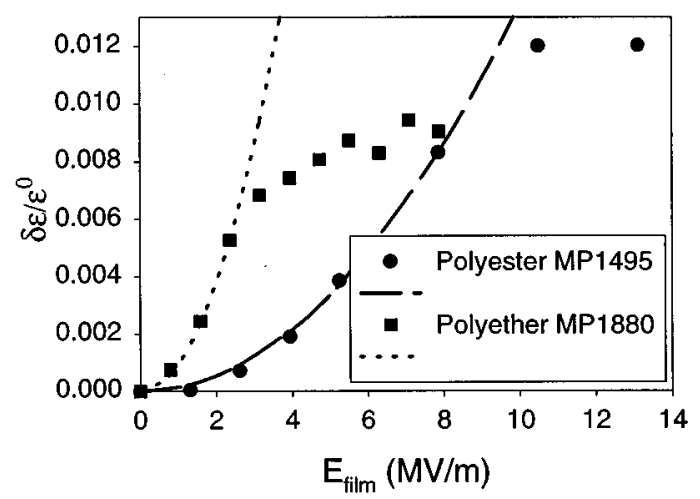

FIG. 5. $\delta \epsilon / \epsilon^{0}$ as a function of electric field strength $\left(E_{\text {film }}=E_{\text {app }}\right)$ for two polyurethane films. The symbols are the experimental data and the curves are parabolic fits to the small field strength data. For the MP1495 data, $\left(\delta \epsilon / \epsilon^{0}\right) / E_{\text {film }}^{2}=1.3 \times 10^{-16}(\mathrm{~m} / \mathrm{V})^{2}$, and for the MP1880 data, $\left(\delta \epsilon / \epsilon^{0}\right) / E_{\text {film }}^{2}=9.5 \times 10^{-16}(\mathrm{~m} / \mathrm{V})^{2}$.

dition to the weight of the top electrode and glass slide, $\approx 80 \mathrm{~g}$ ). Through Eq. (17), these values provide a measure of $\left(d \epsilon_{33} / d u_{33}\right) / \epsilon^{0}$.

$$
\left(-d \epsilon_{33} / d u_{33}\right) / \epsilon^{0}=-\left(a_{1}+a_{2} d u_{k k} / d u_{33}\right) / \epsilon^{0} \quad \text { for }
$$

MP1880 is plotted as a function of the total weight per unit film area, $w$, normalized by the electric pressure scale $\epsilon_{0} E_{\text {film}}^{2} / 2$ in Fig. 6. The ratio of the mechanical and electrical pressures is in the range $0.5-5$, but the polymer deformation remains in the linear elastic region. ${ }^{11}$ The mechanical pressure constrains the film through friction between the polymer film and the electrodes.

The film is relatively nonconstrained when $w$ is small. For a perfectly nonconstrained film, $d u_{k k} / d u_{33}=1-2 \nu \ll 1$, and thus the ordinate in Fig. 6 becomes $-a_{1} / \epsilon^{0}$. As $w$ increases, the friction increases and the film becomes more constrained. For a completely constrained film, $d u_{k k} / d u_{33}=1$, and the ordinate becomes $-\left(a_{1}+a_{2}\right) / \epsilon^{0}$. The data in Fig. 6 appear saturate for large $w$, which we believe corresponds to this large friction limit. Assuming that the limits of small and large $w$ in Fig. 6 do correspond the

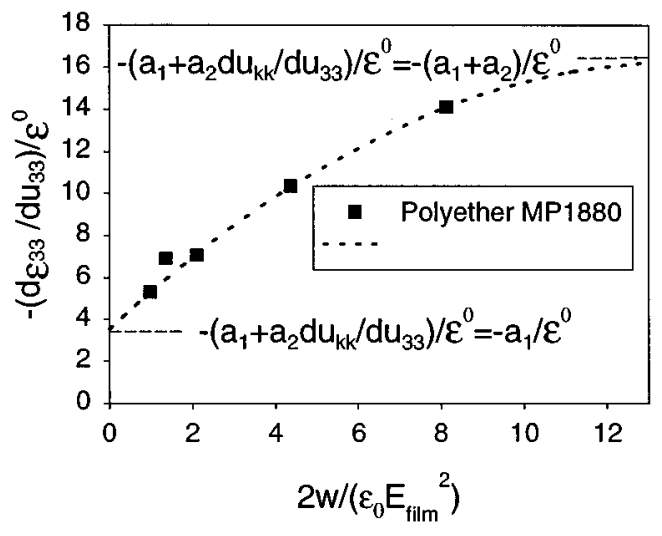

FIG. 6. $\left(-d \epsilon_{33} / d u_{33}\right) / \epsilon^{0}$ as a function of the total weight per unit film area normalized by the electrostatic pressure scale, $2 w / \epsilon_{0} E_{\text {film }}^{2}$ for the MP1880 polyurethane film. All data were taken for a field strength of $4 \mathrm{MV} / \mathrm{m}$. For a nonconstrained film, $2 w / \epsilon_{0} E_{\text {film }}^{2} \rightarrow 0, d \epsilon_{33} / d u_{33}=a_{1} \approx-4 \epsilon^{0}$. For a constrained film, $2 w / \epsilon_{0} E_{\text {film }}^{2} \rightarrow \infty, d \epsilon_{33} / d u_{33}=a_{1}+a_{2} \approx-16 \epsilon^{0}$. 


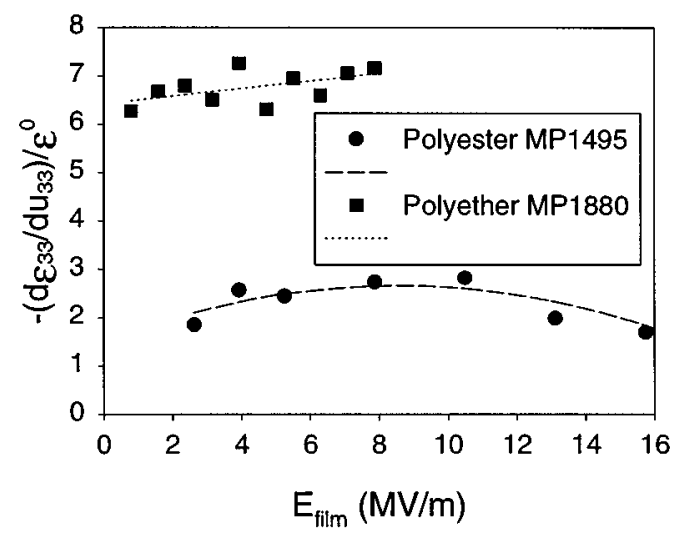

FIG. 7. $\left(-d \epsilon_{33} / d u_{33}\right) / \epsilon^{0}$ as a function of the field strength producing the deformation (with $200 \mathrm{~g}$ placed on the sample capacitor). The derivative remains approximately constant while the electrostatic stresses vary by a factor of about 100. This demonstrates that the experimental procedure for determining the derivative $d \epsilon_{33} / d u_{33}$ through the variations $\delta h$ and $\delta \epsilon$ is correct.

small and large friction limits, respectively, the dielectric coefficients are estimated to be $-a_{1} \approx 4 \epsilon^{0}$, and $-a_{2} \geqslant 12 \epsilon^{0}$. We note that these values are measured at a field frequency of $\approx 1.0 \mathrm{MHz}$.

This method for determining $a_{1}$ and $a_{2}$ is open to criticism because the precise conditions at the polymer-electrode interfaces are unknown. However, we are confident that by adding weights to the capacitor assembly, the friction at the interface increases-therefore, this method does illustrate that by varying $d u_{k k} / d u_{33}$, the relative contributions of $a_{1}$ and $a_{2}$ to $\left(d \epsilon_{33} / d u_{33}\right)$ can be altered and the coefficients themselves extracted.

From the estimates of $a_{1}$ and $a_{2}$ obtained here, two important points follow. First, in contrast to the situation for liquids (e.g., as described by the Clausius-Mossoti model ${ }^{1}$ ), $a_{1} \neq 0$ for the solid polymer studied here. Second, the contribution of $a_{1}+a_{2}$ to the electrostatic force acting to deform the film (see Eqs. (6) and (7)) is more than an order of magnitude larger than the Coulombic attraction between the electrodes - the material's electroactive character arises mainly from $a_{1}$ and $a_{2}$. Ignoring their contribution when estimating electrostatic forces can result in significant error.

The quantity $-\left(d \epsilon_{33} / d u_{33}\right) / \epsilon^{0}=-\left[a_{1}+a_{2}\left(d u_{k k} /\right.\right.$ $\left.\left.d u_{33}\right)\right] / \epsilon^{0}$ is plotted as a function of electric field strength for both polymer films in Fig. 7. Here, $200 \mathrm{~g}$ were placed on the samples (the same weight for all measurements) and the mechanical conditions at the polymer/electrode interfaces were the same for both films. $-\left(d \epsilon_{33} / d u_{33}\right) / \epsilon^{0}$ is different for the two polymers, but in each case remains approximately constant (within experimental uncertainty) over the range of field strengths investigated, which corresponds to a variation of electrostatic stresses of approximately 100 times. This demonstrates that the experimental procedure for determining the derivative $d \epsilon_{33} / d u_{33}$ through the variations $\delta h$ and $\delta \epsilon$ is correct (i.e., $d \epsilon_{33} / d u_{33}$ is accurately determined via Eqs. (15)-(17) over the entire range of field strengths and resulting deformations).

Finally, using the linear model and the parameters estimated here, it is possible to predict electrostrictive deforma- tions under certain conditions. We assume that $\epsilon^{0}=2$, and that $a_{1}$ and $a_{2}$ are independent of frequency. The effective stiffness, $g \approx 7.1 \times 10^{5} \mathrm{~Pa}$, was determined by measuring $\delta h / h^{0}$ due to added weights, without an electric field. The deformation predicted for the MP1880 film is $\left(-\delta h / h^{0}\right) / E_{\text {film }}^{2}=2.1 \times 10^{-16} \mathrm{~m}^{2} / \mathrm{V}^{2}$, using Eqs. (7) and (8). This is $\approx 1.7 \times$ larger than the deformation illustrated in Fig. 4 at small field strengths. We are uncertain about the source of the discrepancy. However, note that if the deformation is assumed to be due only to the Coulombic attraction between the electrodes, i.e., $f_{n}=\epsilon_{0} \epsilon^{0} E_{\text {film }}^{2} / 2$, the prediction would be too small by a factor of about 10 .

\section{CONCLUSIONS}

In this article, we have outlined a linear theory for electrostrictive deformation associated with five material parameters- the Young's modulus, $E_{y}$, Poisson's ratio, $\nu$, the relative dielectric constant in the absence of deformation, $\epsilon^{0}$, and two dielectric coefficients, $a_{1}$ and $a_{2}$, which describe the change in the dielectric tensor with deformation. Determining the deformation requires knowledge of these parameters, as well as appropriate boundary conditions. Deformation and permittivity data for two polymer films is represented well by this linear theory for small to moderate electric field strengths.

A method for measuring the parameters $a_{1}$ and $a_{2}$ has been suggested, based upon measuring the deformation and change in permittivity for at least two different mechanical boundary conditions. We have illustrated such a procedure, where the mechanical boundary conditions are varied by altering the friction at the polymer-electrode interface.

Estimates of the parameters $a_{1}$ and $a_{2}$ suggest that the electrostatic force exerted on a dielectric solid is significantly larger (at least ten times) that that given by the Coulombic attraction between the electrodes alone.

The experimental method illustrated here to measure $a_{1}$ and $a_{2}$ requires excessive speculation about the polymerelectrode interface. Future work will focus on improving the experimental technique to more accurately determine these parameters. Determining the features that control deviations from linearity and the transient responses are also important for applications and will be pursued in future studies.

\section{ACKNOWLEDGMENTS}

The authors thank V. A. Naletova, M. J. Winokur, S. L. Cooper, E. R. Ding, and K. L. Hartley for helpful discussions. This work was supported in part by the Office of Naval Research (Grant No. N00014-94-1-0449) and the National Science Foundation (Grant No. CTS-9502276).

${ }^{1}$ J. A. Stratton, Electromagnetic Theory (McGraw-Hill, New York, 1941).

${ }^{2}$ J. F. Nye, Physical Properties of Crystals (Oxford University, Oxford, 1976).

${ }^{3}$ C. L. Hom and N. Shankar, J. Int. Mat. Syst. Struct. 5, 795 (1994).

${ }^{4}$ K. Elhami, B. Gauthier-Manuel, J. F. Manceau, and F. Bastien, J. Appl. Phys. 77, 3987 (1995).

${ }^{5}$ G. Kloos, J. Phys. D 28, 939 (1995).

${ }^{6}$ L. D. Landau and E. M. Lifshitz, Electrodynamics of Continuous Media (Pergamon, New York, 1984). 
${ }^{7}$ The definitions of $a_{1}$ and $a_{2}$ follow those in Ref. 6. Similar parameters defined in Ref. $8, \widetilde{a_{1}}$ and $\widetilde{a_{2}}$, are related to those employed here by $\widetilde{a_{1}}$ $-\tilde{a_{2}}=a_{1}$ and $\tilde{a_{2}}=a_{2}$.

${ }^{8}$ L. D. Landau and E. M. Lifshitz, Theory of Elasticity (Pergamon, New York, 1970).
${ }^{9}$ J. D. Ferry, Viscoelastic Properties of Polymers (Wiley, New York, 1980). ${ }^{10}$ M. V. Lukashevich, Y. M. Shkel, and I. V. Yaminskii, Magnetohydrodynamics 24, 470 (1989).

${ }^{11}$ P. Wright and P. P. C. Cumming, Solid Polyurethane Elastomers (Gordon and Breach, New York, 1969). 